\title{
Recurrent Implantation Failure and Low Molecular Weight Heparin
}

\author{
Dalia Khalife1, Ghina Ghazeeri² \\ ${ }^{1}$ Department of Obstetrics and Gynecology, American University of Beirut Medical Center, Beirut, Lebanon \\ ${ }^{2}$ Reproductive Endocrinology and Infertility Department, American University of Beirut Medical Center, Beirut, Lebanon \\ Email: khalifehdalia@gmail.com,gg02@aub.edu.lb
}

How to cite this paper: Khalife, D. and Ghazeeri, G. (2018) Recurrent Implantation Failure and Low Molecular Weight Heparin. Open Journal of Obstetrics and Gynecology, 8, 146-162. https://doi.org/10.4236/ojog.2018.82018

Received: January 11, 2017

Accepted: February 9, 2018

Published: February 12, 2018

Copyright $\odot 2018$ by authors and Scientific Research Publishing Inc. This work is licensed under the Creative Commons Attribution International License (CC BY 4.0).

http://creativecommons.org/licenses/by/4.0/

(c) (i) Open Access

\begin{abstract}
Implantation of the embryo into the endometrium is the first step in the establishment of pregnancy. This process is complex, and depends on many factors. Recurrent implantation failure is a source of distress to patients and specialists. It is defined as failure to achieve a viable pregnancy, following " >3 embryo transfers with high quality embryos or the transfer of $\geq 10$ embryos in multiple transfers". Thrombophilic conditions that contribute to recurrent implantation failure are the main concern in this review. The mechanism of implantation failure is believed to be due to decreased blood flow to the endometrium and placenta which can hinder normal endometrial receptivity leading to miscarriage. Defects in early placentation resulting in pregnancy failure, have focused attention on the therapeutic potential of low molecular weight heparin in the implantation process. Heparin has a role at all stages of implantation to improve pregnancy outcomes. There are controversies in literature regarding the association between thrombophilia and recurrent implantation failure and available literature regarding this issue is very heterogeneous. Various investigators, have shown that women with RIF are more likely to have a thrombophilia disorder, yet a clear cause cannot be acknowledged from these studies. Heparin treatment has been evaluated in several studies, showing conflicting evidence. However, several studies have pointed out that it may play a role in a subset of patients who presents a thrombophilia mutation, thus the group of patients that might benefit is needed to be identified. This review is dedicated to evaluate the published literature about the role of low molecular weight heparin in case of recurrent implantation failure with or without the presence of thrombophilia.
\end{abstract}

\section{Keywords}

Heparin, Low-Molecular-Weight Heparin, Recurrent Implantation Failure, Thrombophilia, Anti-Coagulation, Assisted Reproductive Technologies 


\section{Introduction}

While innovations in assisted reproductive technologies have been significant, failure of the viable embryo to implant remains a source of distress to patients and specialists. Defects in early placentation resulting in pregnancy failure, have focused attention on the therapeutic potential of low molecular weight heparin (LMWH) in the implantation process. The clinical evidence supporting the use of LMWH is weak. The literature in this field is filled with challenges, as the published studies are of small sample size, heterogeneous, with different type of biases. This review is dedicated to evaluate the published literature about the role of LMWH in case of recurrent implantation failure (RIF).

\section{Implantation of the Embryo}

Implantation of the embryo into the endometrium is the first step in the establishment of pregnancy. This process is complex, initiated by the recognition and adhesion between the embryo surface and the uterine epithelium, and depends on many factors [1]. It involves, embryo apposition, adhesion, and invasion into the endometrium. First, the embryo attaches to the luminal surface of the endometrium, penetrates the epithelium followed by migration and invasion into the deeper layer of the endometrium to the circulatory system of the mother [2] [3]. Embryo viability and endometrial receptivity are synchronized to allow for successful implantation and establishment of pregnancy [4].

\section{Endometrial Receptivity}

During the menstrual cycle, functional changes in the endometrium are essential for implantation. A variety of secretions from the secretory epithelium are essential for embryo survival such as chemokines, growth factors, and cell adhesion molecules (CAMs) that attract the blastocyst to specific docking sites for implantation.

The embryo implants in the endometrium within a short period of time which is the receptive phase of endometrium or the implantation window that opens 5 to 6 days after ovulation [1]. During this window, the epithelium develops structures known as pinopodes (micro protrusions present on the surface of the endometrium), induced by progesterone, that express chemokines and CAMs that attract the embryo for its adherence [4]. The formation of pinopodes is associated with increased endometrial receptivity. One important mechanism of these pinopodes is the removal of adhesion-inhibiting mucin during the window of implantation, forcing the blastocyst to be in contact with the endometrium [2].

By the third to fourth day after fertilization, the embryo is composed of multiple cells encapsulated by the zona pellucida. At the fifth day after fertilization, the embryo is referred to as a blastocyst which comprises the outer layer of cells known as the trophectoderm, and an inner cell mass, a group of cells lying under the trophectoderm at one end of the blastocele. For a proper implantation, the 
blastocyst should become fixed in position to form a physical contact with the uterus. This developing blastocyst interacts with the endometrial epithelium by producing multiple cytokines, chemokines, and CAMs that facilitate adherence. Any disruption in the synchrony between the blastocyst development and endometrium increases the chances of implantation failure [1] [2] [4].

Understanding the dynamic of the endometrium and the large biochemical and molecular players required for implantation may be helpful to understand the role of LMWH in patients with RIF. Under optimal conditions, the maximal chance of implantation is about $40 \%$ per cycle [2].

A synchronized dialogue between the mother and the developing embryo is essential for an optimal implantation process. The implantation process relies on various chemical factors that facilitate the process of adhesion and invasion. Among these molecules (produced either by the endometrial cells or the blastocyst) are Leukemia inhibiting factor (LIF), Interleukin-1 (IL-1), Heparin-Binding Epidermal Growth Factor (HB-EGF), Prolactin, Insulin-like Growth Factor (IGF-1) and Insulin-like Growth-Factor-Binding Protein (IGFBP-1), adhesion molecules including L-selectin and E-cadherin; extracellular matrix (ECM) proteins, prostaglandins and Bone Morphogenetic Proteins (BMPs) [4] [5].

Three main molecules are involved in the apposition of the embryo: Colonystimulating factor-1 (CSF-1), LIF and their corresponding receptors, are found in human endometrium and preimplantation embryo. IL-1 stimulates trophoblast cells to secrete hCG, endometrium to increase expression of vascular endothelial growth factor (VEGF) and HB-EGF, increasing permeability of capillaries. In return, blastocyst contains receptors that respond to HB-EGF to promote growth and hatching of zona pellucida [2].

As for the adhesion, integrins, selectins and trophinin are involved in this process. Cell adhesion is mediated by expression of laminin and fibronectin from decidualized endometrium and early embryo [2].

Clinically, implantation is considered successful when evidence of intrauterine gestational sac is evident on ultrasound [3]. The implantation rate is defined as the number of intrauterine gestational sac seen by ultrasound divided by the total number of embryos transferred into the uterine cavity. In In Vitro Fertilization (IVF) cycles, the implantation rate is about $25 \%$ at day 3, increasing to $40 \%$ on day $5[3]$.

\section{Implantation Failure (IF)}

IF can refer to two types of situations. Defined "early" when a negative urine or blood pregnancy test is done 10 to 12 days after embryo transfer (no evidence of implantation with no detectable hCG) or "later" following successful migration of the embryo, hCG produced may be detected in the blood or urine but the process is disrupted before an intrauterine sac is visible on ultrasound (evidence of implantation with detectable HCG, biochemical pregnancy) [3]. IF is the result of failed implantation, placentation or compromise of early development [6]. 


\section{Recurrent Implantation Failure (RIF)}

RIF is very frustrating for IVF specialists. It has been determined that half of embryo transfer procedures result in implantation, nonetheless only half of these initiated implantations will result in an ongoing pregnancy [7]. The number of embryos transferred and the number of embryo transfer procedures are taken into account when defining RIF [8].

The 2005 ESHRE PGD Consortium defines RIF as failure to achieve a viable pregnancy, following " $>3$ embryo transfers with high quality embryos or the transfer of $\geq 10$ embryos in multiple transfers; exact numbers to be determined by each center" [9]. Despite many publications on this topic, no unanimous definition exists [10] [11] [12] [13] [14]. And in the era of single embryo transfer, fewer patients will undergo this number of embryo transfer procedures. Therefore, some authors define it as a transfer of at least 4 good-quality embryos in a minimum of 3 fresh or frozen cycles in a woman under the age of 40 years without achieving a clinical pregnancy [3] [15].

Polanski has defined RIF in an expert opinion based on the available literature as the "absence of implantation after two consecutive IVF cycles where the cumulative number of transferred embryos was no less than four for cleavage-stage embryos and no less than two for blastocysts, with all embryos being of good quality and of appropriate developmental stage" [14].

There are many embryo grading systems that embryologists use to evaluate embryos for transfer but a good-quality embryo is defined as having a number of cells corresponding to the day of its development and blastocysts embryos are graded according to the quality of the inner cell mass and surrounding trophectoderm. Thus, recurrent failure may be explained by a uterine pathology, gamete or embryo factors. Patients should be thoroughly worked up to rule out any underlying uterine condition. Multiple treatments are present in the literature aiming to improve embryo quality or endometrial receptivity, however few are evidence based.

Thrombophilic conditions that contribute to RIF is the main concern in this review. This idea came after successfully treating antiphospholipid syndrome with aspirin and LMWH in case of recurrent pregnancy losses [3]. In order to improve cycle outcomes in women with RIF, heparin have been studied. It is anticipated that the mechanism of implantation failure is similar to that of pregnancy loss, with decreased blood flow to the endometrium and placenta which can hinder normal endometrial receptivity leading to miscarriage.

\section{Heparin and Low Molecular Weight Heparin}

The use of heparins has increased since their discovery. In addition to their anticoagulant effects, these molecules have a physiologic role during implantation and placentation and seems to be effective in the prevention and treatment of pregnancy complications [5] [16]. 
Heparin is a polysulphated glycosaminoglycan that interacts with proteins containing positively charged amino acids. Its anticoagulant effect is due to its affinity to anti-thrombin III and catalytic inhibition of factor Xa and thrombin (factor IIa) [5]. LMWH are derived from unfractionated heparin by chemical or enzymatic depolymerization [17]. Demonstrated advantages of LMWH are the greater bioavailability at low doses, the longer half-life, and the more predictable dose response. In addition, LMWH are at least as safe and effective as unfractionated heparin in the treatment of established deep vein thrombosis, but have the advantage that they can be administered once or twice daily without laboratory monitoring [18].

Heparin has a role at all stages of implantation to improve pregnancy outcomes (Figure 1). It has an effect on the expression of adhesion molecules, matrix degrading enzymes and trophoblast apoptosis [5]. Heparin is able to bind to selectin and cadherins molecules, expressed on the blastocyst surface and deciduae, reducing inflammatory cell adhesion, assisting in the blastocyst apposition during the implantation process [19]. In animal models, heparin has been demonstrated to reduces E-cadherin expression, promoting trophoblast invasion and proliferation into the endometrial cells [20].

In a mouse-in-vitro-system, HB-EGF has been demonstrated to promote

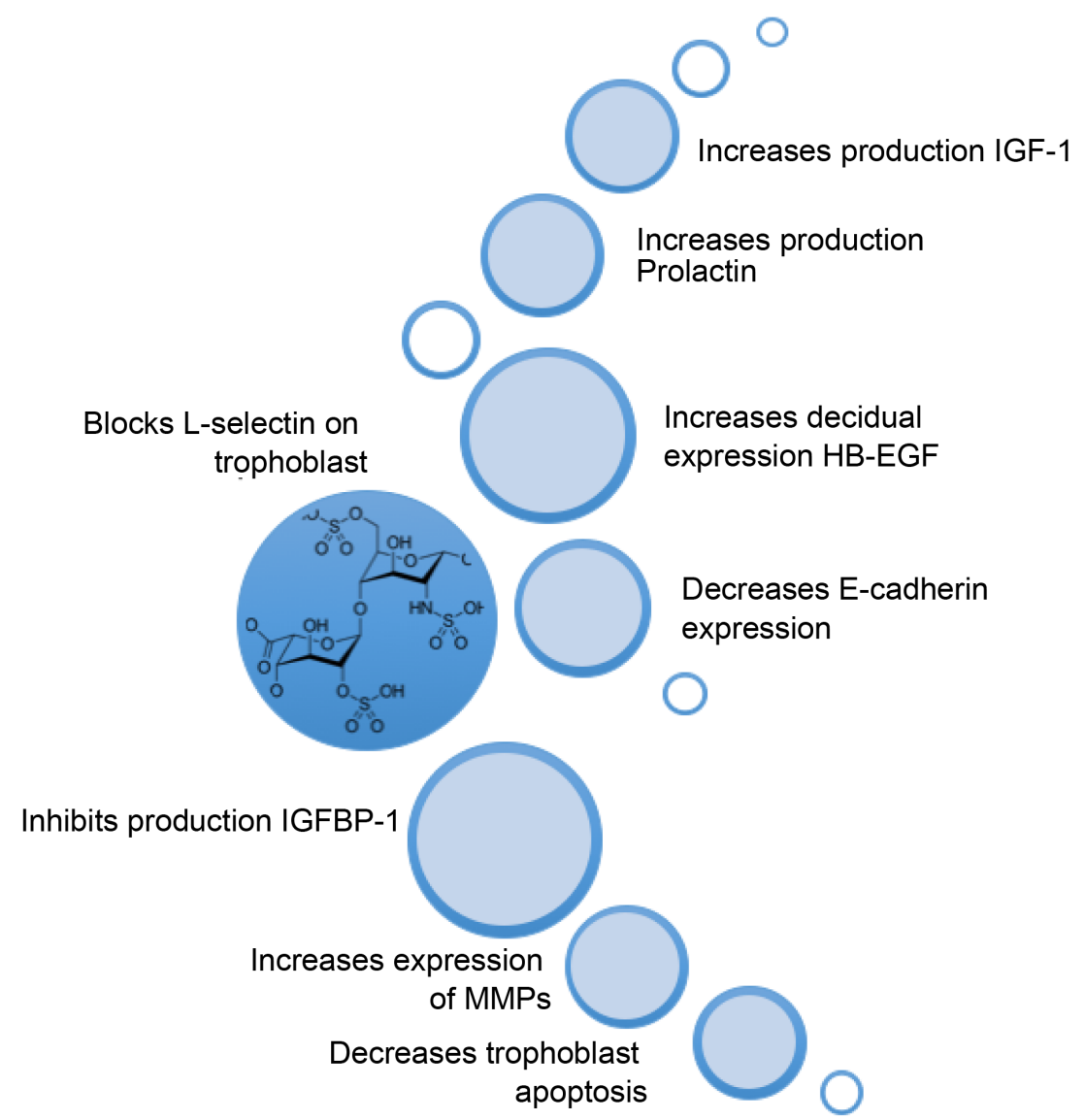

Figure 1. Role of heparin on adhesion, invasion and endometrial receptivity. 
adhesion of the blastocyst to the uterine wall [21] [22]. Heparin induces an increased decidual expression of HB-EGF [23] [24] [25], and induces activation of Activator Protein-1, which regulates the expression of HB-EGF [26]. It also increases the production of both prolactin and IGF-1, inhibits the production of IGFBP-1 that play a role in endometrial receptivity [22] [27]. Interestingly, Hills suggested that heparin inhibits apoptosis in the trophoblasts and attenuates caspase-3 activity. This direct effect acts as survival factor for trophoblast. It induces phosphorylation of EGF receptor exerting an anti-apoptotic signal transduction pathway in human trophoblast, promoting the early stages of embryo development [28].

Even in the absence of thrombophilia, Nelson and Greer, have postulated that heparin in addition to its anticoagulation properties, modulates many of the physiological processes required for blastocyst apposition, adherence, and implantation, to promote establishment of pregnancy [29].

To better understand the impact of heparin on endometrial cells, Fluhr has isolated endometrial cells from hysterectomy specimens which underwent decidualization in vitro and then incubated with unfractionated heparin. Heparin delayed the production of IGFBP-1 and amplified the levels of prolactin and IGF-1 in endometrial cells, thus allowing the decidualization of human endometrial cells and consequently control endometrial receptivity [27].

DiSimone showed that, in patients with antiphospholipid syndrome (APS), immunoglobulin $\mathrm{G}$ affects the invasiveness and differentiation of trophoblast cells after binding to endometrial cells. They were able to show that LMWH reduces the immunoglobulin $\mathrm{G}$ binding to trophoblast cells and restore invasiveness and differentiation [30]. Heparin has been shown also to protect pregnant patients with APS by modulating inflammatory responses and inhibition of complement activation induced by antibodies targeted to decidual tissues rather than by their anticoagulant effects [25] [31].

\section{Thrombophilia and IVF}

Thrombophilia presents a condition of hypercoagulable state. It falls into two categories. Inherited disorders that include factor $\mathrm{V}$ Leiden mutation, methylene tetrahydrofolate reductase (MTHFR) polymorphisms, prothrombin gene mutation, protein $\mathrm{C}$ and $\mathrm{S}$ deficiencies, and antithrombin deficiency. Acquired conditions include anti-phospholipid antibodies syndrome (APA) [32].

It has been suggested that inherited and acquired thrombophilias play a role in abnormal implantation. There are controversies in literature regarding the association between thrombophilia and RIF and available literature regarding this issue is very heterogeneous. APA were the first to be described and studied. Some studies demonstrated significant association [33] [34] [35] [36], and others could not confirm it [37]. Coulam demonstrated that positive APAs were present in $22 \%$ of women with recurrent implantation failure as compared to $5 \%$ in controls [33]. Similarly, several studies have observed an increased prevalence 
of RIF in patients with inherited thrombophilia [15] [38] [39] [40] [41] and others could not verify it [36] [42] [43] [44] [45]. Coulam studied the prevalence of 10 inherited thrombophilia gene mutation in 42 patients with history of RIF in a case-control study. Women with RIF were found to display a significant higher prevalence of PAI-1 4G/5G mutations (38\% vs 10\%) (plasminogen activator inhibitors are inhibitors of plasmin activity hence, fibrinolysis) supporting an abnormal embryo-endometrium dialogue responsible for repeated IVF failures [40].

Qublan, and his colleagues, described a prevalence of combined thrombophilia of $35.6 \%$ in patients with at least three failed IVF cycles and a significant association between incidence of factor V leiden, MTHFR and APA in patients with RIF thus, recommending a universal screening [35]. However, Coughlan reported an overall prevalence of inherited thrombophilia of $11 \%$; and $20 \%$ of patients had positive APA antibodies. Concluding that screening might be routinely ordered only in the case of acquired conditions [36]. Thus, prothrombotic conditions are more prevalent among these patients, yet a clear cause cannot be acknowledged from these studies.

The impact of hemostatic disorders on implantation failure was also investigated with other risk factors such as thyroid disorders. Bellver, included 119 women in a prospective study, and found that combined thrombophilia is higher (19.2\%) in patients with repeated IVF failures, and these patients had a higher prevalence of thyroid autoimmunity [46]. This has given the evidence that thyroid autoimmunity is not only linked to recurrent pregnancy loss but to RIF as well.

A case-control study including 930 patients evaluated the presence of thrombophilia in patients with unexplained infertility, and showed that the odds of inherited thrombophilia was 1.97 significantly higher in patients with unexplained infertility than in the control group suggesting a contribution of thrombophilia by the same mechanism to repeated IVF failures [47]. Two years later, Safdarian matched 96 cases with RIF to controls in which RIF was defined as failure to achieve pregnancy following 2 - 6 IVF cycles where more than 10 high grade embryos transferred into the uterus [48], showed that having at least one inherited thrombophilia is a risk factor for recurrent failure. However, results are limited in view of the nature of the study [41].

The controversial effect of acquired and inherited thrombophilia on repeated failed IVF cycles was evaluated by Di Nisio and his colleagues in a systematic review including 33 studies. They found that women with ART failure test more frequently positive for factor V Leiden and APA compared to fertile women who achieved a livebirth after ART, but this effect was only seen in case-control studies. Cohort studies did not confirm this finding. No significant differences were found in relation to other thrombophilia mutations. The results of this review were inconclusive, since the methodological quality of studies was poor, many were retrospective case-control, known to be limited by poor collection of data 
on potential confounders and recall bias [49]. Ricci has confirmed the absence of association of thrombophilia with adverse IVF outcome by conducting a prospective cohort study of 510 women to compare the prevalence of factor $\mathrm{V}$ leiden and prothrombin gene G20210A mutation in women undergoing IVF and showed no statistical significant difference in the reproductive outcomes (implantation rate (IR), clinical pregnancy rate (CPR), and livebirth rate (LBR)) compared to non-mutation carrier women [43]. These findings were supported also by a retrospective study of 594 women reporting that screening for hypercoagulability is not warranted as it does not improve reproductive outcomes [44], and a cohort study of 70 patients that showed no differences in the incidence of factor $\mathrm{V}$ leiden, prothrombin and MTHFR mutations in patients with repeated failure ( $5.9 \%$ vs $2.8 \%, 0 \%$ vs $5.6 \%$ and $35.3 \%$ vs $50 \%$ ) [45].

Therefore, the value of treatment is still controversial in case of patients testing positive for these antibodies.

\section{Thrombophilia, Heparin and IVF}

1) Acquired thrombophilia

Various investigators, have shown that women with RIF are more likely to have a thrombophilia disorder that is explained by microthrombosis formation at the implantation site, impairing the invasion of maternal vessels by the trophoblast, leading to implantation failure [50]. Heparin treatment has been evaluated in several studies, showing conflicting evidence, however most were observational in nature. Six studies were conducted on patients with RIF and acquired thrombophilia, positive APA treated with heparin. Two of these studies were observational, and have shown that heparin and aspirin do not improve pregnancy rates [51] [52]. This finding was replicated in a randomized prospective study on 143 patients by Stern, showing that heparin and aspirin did not improve pregnancy or implantation rates for patients with acquired thrombophilia and RIF [53]. The other three studies recommended that any women undergoing IVF should be tested for acquired thrombophilia prior to initiating ovarian stimulation, and those with positive antibodies should be treated with heparin with or without low-dose aspirin [15] [54] [55].

Qublan et al., have demonstrated the effect of heparin on a population of patients with three or more failed IVF attempts and at least one thrombophilia disorder. They reported significantly higher IR, CPR and LBR compared with placebo in a placebo-controlled, randomized trial (20.9\% vs $6.1 \%, 31 \%$ vs. $9.6 \%$, $23.8 \%$ vs $2.8 \%$ respectively; $\mathrm{p}<0.001, \mathrm{p}<0.05, \mathrm{p}<0.05$, respectively). They also showed a higher miscarriage rate in the placebo-treated group and very few complications between the 2 study groups suggesting effectiveness and safety of heparin [15].

2) Inherited thrombophilia

In a retrospective analysis including data of 265 patients, the role of LMWH was evaluated in RIF after screening for inherited thrombophilia. They found 
significantly higher CPR in patients treated with LMWH (29.52\% vs $17.19 \%)$ [56].

Lattova in 2015, showed consistent results, where patients with RIF should be tested for thrombophilia mutations and LMWH should be considered individually. The use of treatment is only relevant for patients with established acquired or congenital thrombophilia disorder [1]. These data speculate that heparin has a role in improving implantation by enhancing endometrial receptivity and decidualization rather than by their anticoagulant effect.

3) No evidence of thrombophilia

The effect of heparin in case of RIF on reproductive outcomes has been studied in the absence of thrombophilia where 4 randomized controlled studies (RCTs) were conducted. Urman et al., in a randomized controlled pilot study of 150 patients with 2 or more failed IVF cycles, used heparin in patients with RIF in patients with no evidence of thrombophilia. Heparin group showed a relative increase by $30 \%$ in LBR, however statistical significance was not reached due to the low statistical power and the small sample size of the study [57]. However, a similar randomized control study of 172 women under 40 years of age failed to show also any clinical improvement in the LMWH group (IR, CPR, LBR in the LMWH group compared to controls were respectively $15 \%$ vs $12 \%, 26 \%$ vs $20 \%$, $21 \%$ vs $16 \%$ ) [58]. The same data was replicated in a larger single-center study on 219 women with no coagulation disorders who received LMWH at oocyte retrieval and showed that CPR, LBR, and IR were higher in the LMWH group $(35.4 \%, 31.2 \%, 21.1 \%)$ compared to control group $(27.9 \%, 23.2 \%, 15.8 \%)$ but failed to show a significant difference in outcomes. This study was subject to significant bias since allocation of treatment was dependent on clinician preference and it was ended before achieving the sample size needed to detect a difference [25]. Recently, Hamdi randomized 100 patients with history of 2 or more failures, no significant differences in IR were noted between the 2 groups (32\% vs $30 \%)$ [59].

A recent meta-analysis with systematic review by Potdar compared the use of LMWH with placebo in women with RIF undergoing IVF and evaluated its effect on LBR and IR [60]. They included only three randomized controlled prospective studies which used heparin as the only intervention, started after oocyte retrieval or from the day of embryo transfer. Two out of the three RCTs included patients with RIF without evidence of thrombophilia. They concluded a significant improvement in LBR $(R R=1.79, \mathrm{p}=0.02)$, however the IR showed a non-significant trend toward improvement $(R R=1.73, p=0.06)$. And when pooling the results of studies with only RIF not associated with thrombophilia, significant results were not reached [60]. It is worth mentioning some shortcomings in these studies. All three studies were unclear for detection bias, in which the study population included was not homogeneous, as these studies had different definitions for RIF. Berker and Urman defined RIF by failure of two consecutive Intra Cytoplasmic Sperm Injection (ICSI) cycles [25] [57], whereby Qublan included patients with $\geq 3$ previous IVF failures and a known thrombo- 
philia (inherited or acquired) [15]. In view of the clinical heterogeneity in study population regarding RIF definition and the absence or presence of thrombophilia, results should not be generalized and interpreted with caution. However, this systematic review has its own strengths in which patients in the three studies had almost similar baseline characteristics, similar intervention and considered same outcome measures. The authors of this meta-analyses concluded that LMWH has the potential to facilitate implantation by enhancing endometrial receptivity and decidualization in addition to its anticoagulant effect [60].

Another comprehensive review published previously where 10 studies were analyzed, focused on the effect of heparin on IVF outcome. After pooling the results, randomized studies showed no difference in the CPR (RR 1.23, 95\% CI 0.97 - 1.57), LBR (RR 1.27, 95\% CI 0.89 - 1.81) and IR (RR 1.39, 95\% CI 0.96 2.01 ) and abortion rate (RR $0.77,95 \%$ CI $0.24-2.42$ ). However, this review was highly susceptible to publication bias secondary to the high methodological heterogeneity between the trials in which heparin's effect was assessed irrespective of the failed cycles, small numbers of women included with considerable difference in the administered formulations, dosing, timing of onset and duration of therapy [61].

A systematic review and meta-analysis by Dentali, included 3 RCTs comparing LMWH with placebo in 305 women with [15] and without known thrombophilia undergoing ICSI and the effect of heparin on CPR and LBR was investigated [57] [58]. It showed a higher CPR (RR 1.38, 95\% CI 1.02 - 1.87) and LBR (RR 1.54, 95\% CI 1.05 - 2.26) among patients who used LMWH. However, when repeating the analyses with random effect model, an approach that takes into account variance among studies, differences were no longer statistically significant [62].

The previous three meta-analyses, including a Cochrane review, used LBR as primary outcome. All the individual studies included were small, highly heterogeneous in terms of inclusion and exclusion criteria with poor overall methodology [60] [61] [62]. However, despite the fact that the effect of heparin as an adjuvant treatment in case of RIF requires further evaluation with high-quality powered studies, we can conclude that the meta-analyses by Potdar has a more consistent study population with patients having more than 3 RIF and its results can be used to provide evidence-based information in case of RIF.

\section{Future Perspectives}

Since, many researchers have evaluated the physiological processes required for blastocyst apposition, adherence and implantation, other factors are now investigated.

A French team published recently a prospective longitudinal study aiming to identify biomarkers of hypercoagulability that can have a predictive value for failed IVF outcome. Procoagulant substances are related to infertility. The decidualized human endometrial stromal cells express tissue factor, stimulates the 
production of thrombin which is required for cell proliferation, neoangiogenesis and invasion of trophoblast. This shift in blood coagulation by enhancement in thrombin generation have a positive effect on positive IVF outcome. Biomarkers included Thrombin generation, procoagulant phospholipid clotting time (procoag PPL CT), thrombomodulin, tissue factor activity, factor VIII, von Willebrand factor, D-Dimers and fibrinogen.

Patients were found to have hypercoagulable state at the baseline, before any treatment and this state is not modified by any hormonal treatment. Among these biomarkers, Procoag-PPL CT (shortened), D-Dimeres (increased) and thrombin (decreased) are the most clinically relevant for identifying patients who are at risk of IVF failure. Their results are in accordance with previous study who proposed that the increase of D-Dimers levels is a predictor of IVF failure [63]. However, the sample size was small and does not allow generalizability of results [64]. The results of this study need to be validated in larger powered studies, to optimize IVF efficiency.

Another possible risk factor of RIF that is growing tremendously and diagnostic procedures are implemented in order to improve this condition is now on cMPs, circulating cell-derived microparticles known to be associated with multiple prothrombotic states. cMPs play a central role in cell signaling, inflammation, thrombosis and vascular function, therefore are highly thrombogenic. They are implicated in cellular activation and apoptosis. It has been shown that cMPs affect trophoblast migration and invasion. They can be assessed using a readily available commercial functional assay and were evaluated by several studies in recurrent spontaneous abortion and were shown to exist in high levels. It has been postulated then, that patients with RIF may have elevated cMP count as the case in spontaneous abortion. This case-control study showed that cMP is significantly associated with patients with RIF as well (RIF group: $15.8 \pm 6.2 \mathrm{nM}$ PS eq; $\mathrm{p}<0.05$ ), provided new data on the pathogenesis of RIF and increased cMP may be a marker of chronic state of inflammation and coagulation [65]. However, further studies are needed to be conducted to study the effect of heparin on cMPs.

\section{Conclusion}

This review aimed to focus on the effect of heparin on the physiologic process of implantation and its use as an adjuvant treatment in patients with RIF with or without the presence of thrombophilia. The evidence on the effectiveness of heparin remains conflicting because of the lack of knowledge and considerable heterogeneity in the studies. Further research and powered larger RCTs are needed, as there could be a potential role of heparin in case of repeated failures of implantation. On the basis of published literature, there's no evidence to conclude that LMWH can be prescribed as empirical treatment in patients who have experienced multiple implantation failures, however, several studies have pointed out that it may play a role in a subset of patients who presents a throm- 
bophilia mutation, thus the group of patients that might benefit is needed to be identified. Another important issue to be determined is the period of administration, at or after egg collection or at embryo transfer.

In view of the psychological, emotional and financial distress, it remains a draining clinical problem for infertile couples and physicians as well.

\section{Conflict of Interest Statement}

The authors declare that the research was conducted in the absence of any commercial or financial relationships that could be taken as a potential conflict of interest.

\section{References}

[1] Lattova, V., Dostal, J., Pešková, M., Sobek Jr., A. and Procházka, M. (2015) Recurrent Implantation Failure and Thrombophilia. Ceska Gynekologie, 80, 5-10.

[2] Fritz, M.A. and Speroff, L. (2010) Sperm and Egg Transport, Fertilization, and Implantation, Clinical Gynecologic Endocrinology and Infertility, Wolters Kluwer Health.

[3] Coughlan, C., Ledger, W., Wang, Q., Liu, F., Demirol, A., Gurgan, T., et al. (2014) Recurrent Implantation Failure: Definition and Management. Reproductive Biomedicine Online, 28, 14-38. https://doi.org/10.1016/j.rbmo.2013.08.011

[4] Mesiano, S. The Endocrinology of Human Pregnancy and Fetal-Placental Neuroendocrine Development, Yen \& Jaffe's Reproductive Endocrinology, Chapter 12. 243-271.

[5] Quaranta, M., Erez, O., Mastrolia, S.A., Koifman, A., Leron, E., Eshkoli, T., et al. (2015) The Physiologic and Therapeutic Role of Heparin in Implantation and Placentation. PeerJ, 3, e691. https://doi.org/10.7717/peerj.691

[6] Bates, S.M. (2014) Anticoagulation and In Vitro Fertilization and Ovarian Stimulation. ASH Education Program Book, 2014, 379-386.

[7] Boomsma, C.M., Kavelaars, A., Eijkemans, M.J.C., Lentjes, E.G., Fauser, B.C.J.M., Heijnen, C.J. and Macklon, N.S. (2009) Endometrial Secretion Analysis Identifies a Cytokine Profile Predictive of Pregnancy in IVF. Human Reproduction, 24, 1427-1435. https://doi.org/10.1093/humrep/dep011

[8] Timeva, T., Shterev, A. and Kyurkchiev, S. (2014) Recurrent Implantation Failure: The Role of the Endometrium. Journal of Reproduction \& Infertility, 15, 173.

[9] Thornhill, A.R., deDie-Smulders, C.E., Geraedts, J.P., Harper, J.C., Harton, G.L., Lavery, S.A., et al. (2005) ESHRE PGD Consortium "Best Practice Guidelines for Clinical Preimplantation Genetic Diagnosis (PGD) and Preimplantation Genetic Screening (PGS)". Human Reproduction, 20, 35-48.

https://doi.org/10.1093/humrep/deh579

[10] Das, M. and Holzer, H.E. (2012) Recurrent Implantation Failure: Gamete and Embryo Factors. Fertility and Sterility, 97, 1021-1027. https://doi.org/10.1016/j.fertnstert.2012.02.029

[11] Laufer, N. and Simon, A. (2012) Recurrent Implantation Failure: Current Update and Clinical Approach to an Ongoing Challenge. Fertility and Sterility, 97, 1019-1020. https://doi.org/10.1016/j.fertnstert.2012.03.033

[12] Penzias, A.S. (2012) Recurrent IVF Failure: Other Factors. Fertility and Sterility, 97, 
1033-1038.

[13] Urman, B., Yakin, K. and Balaban, B. (2005) Recurrent Implantation Failure in Assisted Reproduction: How to Counsel and Manage. A. General Considerations and Treatment Options that May Benefit the Couple. Reproductive Biomedicine Online, 11, 371-381. https://doi.org/10.1016/S1472-6483(10)60846-2

[14] Polanski, L.T., Baumgarten, M.N., Quenby, S., Brosens, J., Campbell, B.K. and Raine-Fenning, N.J. (2014) What Exactly Do We Mean by "Recurrent Implantation Failure"? A Systematic Review and Opinion. Reproductive Biomedicine Online, 28, 409-423. https://doi.org/10.1016/j.rbmo.2013.12.006

[15] Qublan, H., Amarin, Z., Dabbas, M., Farraj, A.E., Beni-Merei, Z., Al-Akash, H., Al-Ahmad, N., et al. (2008) Low-Molecular-Weight Heparin in the Treatment of Recurrent IVF-ET Failure and Thrombophilia: A Prospective Randomized Placebo-Controlled Trial. Human Fertility, 11, 246-253.

https://doi.org/10.1080/14647270801995431

[16] Romero, R., Dekker, G., Kupferminc, M., Saade, G., Livingston, J., Peaceman, A., Gomez, R., et al. (2002) Can Heparin Prevent Adverse Pregnancy Outcome? The Journal of Maternal-Fetal \& Neonatal Medicine, 12, 1-8. https://doi.org/10.1080/jmf.12.1.1.8

[17] Greer, I. and Hunt, B.J. (2005) Low Molecular Weight Heparin in Pregnancy: Current Issues. British Journal of Haematology, 128, 593-601. https://doi.org/10.1111/j.1365-2141.2004.05304.x

[18] Shriver, Z., Sundaram, M., Venkataraman, G., Fareed, J., Linhardt, R., Biemann, K. and Sasisekharan, R. (2000) Cleavage of the Antithrombin III Binding Site in Heparin by Heparinases and Its Implication in the Generation of Low Molecular Weight Heparin. Proceedings of the National Academy of Sciences, 97, 10365-10370. https://doi.org/10.1073/pnas.97.19.10365

[19] Stevenson, J.L., Choi, S.H. and Varki, A. (2005) Differential Metastasis Inhibition by Clinically Relevant Levels of Heparins-Correlation with Selectin Inhibition, not Antithrombotic Activity. Clinical Cancer Research, 11, 7003-7011. https://doi.org/10.1158/1078-0432.CCR-05-1131

[20] Tersigni, C., Marana, R., Santamarìa, A., Castellani, R., Scambia, G. and Simone, N.D. (2012) In vitro Evidences of Heparin's Effects on Embryo Implantation and Trophoblast Development. Reproductive Sciences, 19, 454-462. https://doi.org/10.1177/1933719111430994

[21] Raab, G. and Klagsbrun, M. (1997) Heparin-Binding EGF-Like Growth Factor. Biochimica et Biophysica Acta (BBA)-Reviews on Cancer, 1333, F179-F199. https://doi.org/10.1016/S0304-419X(97)00024-3

[22] Tamada, H., Higashiyama, C., Takano, H., Kawate, N., Inaba, T. and Sawada, T. (1999) The Effects of Heparin-Binding Epidermal Growth Factor-Like Growth Factor on Preimplantation-Embryo Development and Implantation in the Rat. Life Sciences, 64, 1967-1973. https://doi.org/10.1016/S0024-3205(99)00128-9

[23] Di Simone, N., Di Nicuolo, F., Castellani, R., Veglia, M., Tersigni, C., Silano, M. and Marana, R. (2012) Low-Molecular-Weight Heparins Induce Decidual Heparin-Binding Epidermal Growth Factor-Like Growth Factor Expression and Promote Survival of Decidual Cells Undergoing Apoptosis. Fertility and Sterility, 97, 169-177. https://doi.org/10.1016/j.fertnstert.2011.10.021

[24] Bohlmann, M.K. (2011) Effects and Effectiveness of Heparin in Assisted Reproduction. Journal of Reproductive Immunology, 90, 82-90.

https://doi.org/10.1016/j.jri.2011.03.004 
[25] Berker, B., Taşkın, S., Kahraman, K., Taşkın, E.A., Atabekoğlu, C. and Sönmezer, M. (2011) The Role of Low-Molecular-Weight Heparin in Recurrent Implantation Failure: A Prospective, Quasi-Randomized, Controlled Study. Fertility and Sterility, 95, 2499-2502. https://doi.org/10.1016/j.fertnstert.2010.12.033

[26] D’Ippolito, S., Marana, R., Di Nicuolo, F., Castellani, R., Veglia, M., Stinson, J. and Di Simone, N. (2012) Effect of Low Molecular Weight Heparins (LMWHs) on Antiphospholipid Antibodies (aPL)-Mediated Inhibition of Endometrial Angiogenesis. PLoS ONE, 7, e29660. https://doi.org/10.1371/journal.pone.0029660

[27] Fluhr, H., Spratte, J., Ehrhardt, J., Steinmüller, F., Licht, P. and Zygmunt, M. (2010) Heparin and Low-Molecular-Weight Heparins Modulate the Decidualization of Human Endometrial Stromal Cells. Fertility and Sterility, 93, 2581-2587. https://doi.org/10.1016/j.fertnstert.2009.10.025

[28] Hills, F.A., Abrahams, V.M., González-Timón, B., Francis, J., Cloke, B., Hinkson, L. and Lam, E.W.F. (2006) Heparin Prevents Programmed Cell Death in Human Trophoblast. MHR: Basic Science of Reproductive Medicine, 12, 237-243.

[29] Nelson, S.M. and Greer, I.A. (2008) The Potential Role of Heparin in Assisted Conception. Human Reproduction Update, 14, 623-645.

https://doi.org/10.1093/humupd/dmn031

[30] DiSimone, N., Caliandro, D., Castellani, R., Ferrazzani, S., DeCarolis, S. and Caruso, A. (1999) Low-Molecular Weight Heparin Restores in-vitro Trophoblast Invasiveness and Differentiation in Presence of Immunoglobulin G Fractions Obtained from Patients with Antiphospholipid Syndrome. Human Reproduction, 14, 489-495. https://doi.org/10.1093/humrep/14.2.489

[31] Girardi, G., Redecha, P. and Salmon, J.E. (2004) Heparin Prevents Antiphospholipid Antibody-Induced Fetal Loss by Inhibiting Complement Activation. Nature Medicine, 10, 1222-1226. https://doi.org/10.1038/nm1121

[32] Bick, R.L. (2006) Hereditary and Acquired Thrombophilic Disorders. Clinical and Applied Thrombosis/Hemostasis, 12, 125-135. https://doi.org/10.1177/107602960601200201

[33] Coulam, C.B., Kaider, B.D., Kaider, A.S., Janowicz, P. and Roussev, R.G. (1997) Antiphospholipid Antibodies Associated with Implantation Failure after IVF/ET. Journal of Assisted Reproduction and Genetics, 14, 603-608. https://doi.org/10.1023/A:1022588903620

[34] Stern, C., Chamley, L., Hale, L., Kloss, M., Speirs, A. and Baker, H.G. (1998) Antibodies to $\beta_{2}$ Glycoprotein I Are Associated with in vitro Fertilization Implantation Failure as Well as Recurrent Miscarriage: Results of a Prevalence Study. Fertility and Sterility, 70, 938-944. https://doi.org/10.1016/S0015-0282(98)00312-4

[35] Qublan, H.S., Eid, S.S., Ababneh, H.A., Amarin, Z.O., Smadi, A.Z., Al-Khafaji, F.F. and Khader, Y.S. (2006) Acquired and Inherited Thrombophilia: Implication in Recurrent IVF and Embryo Transfer Failure. Human Reproduction, 21, 2694-2698. https://doi.org/10.1093/humrep/del203

[36] Coughlan, C., Yuan, X., Nafee, T., Yan, J., Mariee, N. and Li, T.C. (2013) The Clinical Characteristics of Women with Recurrent Implantation Failure. Journal of $O b$ stetrics and Gynaecology, 33, 494-498. https://doi.org/10.3109/01443615.2013.782280

[37] Hornstein, M.D., Davis, O.K., Massey, J.B., Paulson, R.J. and Collins, J.A. (2000) Antiphospholipid Antibodies and in vitro Fertilization Success: A Meta-Analysis. Fertility and Sterility, 73, 330-333. https://doi.org/10.1016/S0015-0282(99)00498-7

[38] Azem, F., Many, A., Yovel, I., Amit, A., Lessing, J.B. and Kupferminc, M.J. (2004) 
Increased Rates of Thrombophilia in Women with Repeated IVF Failures. Human Reproduction, 19, 368-370. https://doi.org/10.1093/humrep/deh069

[39] Grandone, E., Colaizzo, D., Bue, A.L., Checola, M.G., Cittadini, E. and Margaglione, M. (2001) Inherited Thrombophilia and in vitro Fertilization Implantation Failure. Fertility and Sterility, 76, 201-202. https://doi.org/10.1016/S0015-0282(01)01832-5

[40] Coulam, C.B., Jeyendran, R.S., Fishel, L.A. and Roussev, R. (2006) Multiple Thrombophilic Gene Mutations Are Risk Factors for Implantation Failure. Reproductive Biomedicine Online, 12, 322-327. https://doi.org/10.1016/S1472-6483(10)61004-8

[41] Safdarian, L., Najmi, Z., Aleyasin, A., Aghahosseini, M., Rashidi, M. and Asadollah, S. (2014) Recurrent IVF Failure and Hereditary Thrombophilia. Iranian Journal of Reproductive Medicine, 12, 467-470.

[42] Simur, A., Özdemir, S., Acar, H., Çolakoğlu, M.C., Görkemli, H., Balcı, O. and Nergis, S. (2009) Repeated in vitro Fertilization Failure and Its Relation with Thrombophilia. Gynecologic and Obstetric Investigation, 67, 109-112. https://doi.org/10.1159/000165776

[43] Ricci, G., Bogatti, P., Fischer-Tamaro, L., Giolo, E., Luppi, S., Montico, M. and Morgutti, M. (2011) Factor V Leiden and Prothrombin Gene G20210A Mutation and in vitro Fertilization: Prospective Cohort Study. Human Reproduction, 26, 3068-3077. https://doi.org/10.1093/humrep/der261

[44] Steinvil, A., Raz, R., Berliner, S., Steinberg, D.M., Zeltser, D., Levran, D. and Salomon, O. (2012) Association of Common Thrombophilias and Antiphospholipid Antibodies with Success Rate of in vitro Fertilisation. Thrombosis and Haemostasis, 108, 1192-1197. https://doi.org/10.1160/TH12-06-0381

[45] Hamdi, K., Vaezi, M., Dagigazar, B., Sadagiani, M.M., Farzadi, L. and Pashaei-Asl, M. (2012) Association between Thrombophilia and Repeated Assisted Reproductive Technology Failures. Advanced Pharmaceutical Bulletin, 2, 233.

[46] Bellver, J., Soares, S.R., Álvarez, C., Muñoz, E., Ramírez, A., Rubio, C. and Pellicer, A. (2007) The Role of Thrombophilia and Thyroid Autoimmunity in Unexplained Infertility, Implantation Failure and Recurrent Spontaneous Abortion. Human Reproduction, 23, 278-284. https://doi.org/10.1093/humrep/dem383

[47] Fatini, C., Conti, L., Turillazzi, V., Sticchi, E., Romagnuolo, I., Milanini, M.N. and Noci, I. (2012) Unexplained Infertility: Association with Inherited Thrombophilia. Thrombosis Research, 129, e185-e188. https://doi.org/10.1016/j.thromres.2012.02.012

[48] Tan, B.K., Vandekerckhove, P., Kennedy, R. and Keay, S.D. (2005) Investigation and Current Management of Recurrent IVF Treatment Failure in the UK. BJOG: An International Journal of Obstetrics \& Gynaecology, 112, 773-780. https://doi.org/10.1111/j.1471-0528.2005.00523.x

[49] Di Nisio, M., Rutjes, A.W., Ferrante, N., Tiboni, G.M., Cuccurullo, F. and Porreca, E. (2011) Thrombophilia and Outcomes of Assisted Reproduction Technologies: A Systematic Review and Meta-Analysis. Blood, 118, 2670-2678.

https://doi.org/10.1182/blood-2011-03-340216

[50] Geva, E., Amit, A., Lerner-Geva, L., Azem, F., Yovel, I. and Lessing, J.B. (1995) Autoimmune Disorders: Another Possible Cause for in-vitro Fertilization and Embryo Transfer Failure. Human Reproduction, 10, 2560-2563. https://doi.org/10.1093/oxfordjournals.humrep.a135745

[51] Schenk, L.M., Butler, L., Morris, J.P., Cox, B., Lecte, J., Abuhamed, A. and Muasher, S. (1996) Heparin and Aspirin Treatment Yields Higher Implantation Rates in IVF 
Patients with Antiphospholipid Antibody Seropositivity Compared to Untreated Seronegative Patients. Fifty-Second Annual Meeting of the American Society for Reproductive Medicine, Boston, MA, 2-6 November 1996, 2-6.

[52] Kutteh, W.H. (1997) Antiphospholipid Antibodies and Reproduction. Journal of Reproductive Immunology, 35, 151-171. https://doi.org/10.1016/S0165-0378(97)00059-4

[53] Stern, C., Chamley, L., Norris, H., Hale, L. and Baker, H.G. (2003) A Randomized, Double-Blind, Placebo-Controlled Trial of Heparin and Aspirin for Women with in vitro Fertilization Implantation Failure and Antiphospholipid or Antinuclear Antibodies. Fertility and Sterility, 80, 376-383. https://doi.org/10.1016/S0015-0282(03)00610-1

[54] Sher, G., Feinman, M., Zouves, C., Kuttner, G., Maassarani, G., Salem, R. and Chong, P. (1994) Immunology: High Fecundity Rates Following in-vitro Fertilization and Embryo Transfer in Antiphospholipid Antibody Seropositive Women Treated with Heparin and Aspirin. Human Reproduction, 9, 2278-2283. https://doi.org/10.1093/oxfordjournals.humrep.a138437

[55] Sher, G., Zouves, C., Maassarani, G., Feinman, M., Matzner, W., Chong, P. and Ching, W. (1998) A Rational Basis for the Use of Combined Heparin/Aspirin and IVIG Immunotherapy in the Treatment of Recurrent IVF Failure Associated with Antiphospholipid Antibodies. American Journal of Reproductive Immunology, 39, 391-394. https://doi.org/10.1111/j.1600-0897.1998.tb00375.x

[56] Lodigiani, C., Di Micco, P., Ferrazzi, P., Librè, L., Arfuso, V., Polatti, F. and Brenner, B. (2011) Low-Molecular-Weight Heparin in Women with Repeated Implantation Failure. Women's Health, 7, 425-431. https://doi.org/10.2217/WHE.11.38

[57] Urman, B., Ata, B., Yakin, K., Alatas, C., Aksoy, S., Mercan, R. and Balaban, B. (2009) Luteal Phase Empirical Low Molecular Weight Heparin Administration in Patients with Failed ICSI Embryo Transfer Cycles: A Randomized Open-Labeled Pilot Trial. Human Reproduction, 24, 1640-1647.

https://doi.org/10.1093/humrep/dep086

[58] Noci, I., Milanini, M.N., Ruggiero, M., Papini, F., Fuzzi, B. and Artini, P.G. (2011) Effect of Dalteparin Sodium Administration on IVF Outcome in Non-Thrombophilic Young Women: A Pilot Study. Reproductive Biomedicine Online, 22, 615-620. https://doi.org/10.1016/j.rbmo.2011.03.016

[59] Hamdi, K., Danaii, S., La'ya Farzadi, S.A., Chalabizadeh, A. and Sabet, S.A. (2015) The Role of Heparin in Embryo Implantation in Women with Recurrent Implantation Failure in the Cycles of Assisted Reproductive Techniques (Without History of Thrombophilia). Journal of Family \& Reproductive Health, 9, 59-64.

[60] Potdar, N., Gelbaya, T.A., Konje, J.C. and Nardo, L.G. (2013) Adjunct Low-Molecular-Weight Heparin to Improve Live Birth Rate after Recurrent Implantation Failure: A Systematic Review And Meta-Analysis. Human Reproduction Update, 19, 674-684. https://doi.org/10.1093/humupd/dmt032

[61] Seshadri, S., Sunkara, S.K., Khalaf, Y., El-Toukhy, T. and Hamoda, H. (2012) Effect of Heparin on the Outcome of IVF Treatment: A Systematic Review and Meta-Analysis. Reproductive Biomedicine Online, 25, 572-584. https://doi.org/10.1016/j.rbmo.2012.08.007

[62] Dentali, F., Grandone, E., Rezoagli, E. and Ageno, W. (2011) Efficacy of Low Molecular Weight Heparin in Patients Undergoing in vitro Fertilization or Intracytoplasmic Sperm Injection. Journal of Thrombosis and Haemostasis, 9, 2503-2506. https://doi.org/10.1111/j.1538-7836.2011.04535.x 
[63] Di Nisio, M., Porreca, E., Di Donato, V. and Tiboni, G.M. (2014) Plasma Concentrations of D-Dimer and Outcome of in vitro Fertilization. Journal of Ovarian Research, 7, 58. https://doi.org/10.1186/1757-2215-7-58

[64] Gerotziafas, G.T., Van Dreden, P., d’Argent, E.M., Lefkou, E., Grusse, M., Comtet, M. and Elalamy, I. (2017) Impact of Blood Hypercoagulability on in vitro Fertilization Outcomes: A Prospective Longitudinal Observational Study. Thrombosis Journal, 15, 9. https://doi.org/10.1186/s12959-017-0131-7

[65] Martínez-Zamora, M.A., Tàssies, D., Reverter, J.C., Creus, M., Casals, G., Cívico, S. and Balasch, J. (2016) Increased Circulating Cell-Derived Microparticle Count Is Associated with Recurrent Implantation Failure after IVF and Embryo Transfer. Reproductive Biomedicine Online, 33, 168-173.

https://doi.org/10.1016/j.rbmo.2016.05.005

\section{Abbreviation List}

RIF: Recurrent Implantation Failure

LMWH: Low Molecular Weight Heparin 